Foyle, D.C., Ahumada, A.J., Larimer, J. and Sweet, B.T. (1992). Enhanced/synthetic vision systems: Human factors research and implications for future systems. SAE Transactions: Journal of Aerospace, 101, 1734-1741.

\title{
Enhanced/Synthetic Vision Systems: Human Factors Research and Implications for Future Systems
}

\author{
David C. Foyle, Albert J. Ahumada, James Larimer and Barbara Townsend Sweet \\ Aerospace Human Factors Research Division (MS 262-3) \\ NASA Ames Research Center \\ Moffett Field, CA 94035-1000
}

\begin{abstract}
This paper reviews recent human factors research studies conducted in the Aerospace Human Factors Research Division at NASA Ames Research Center related to the development and usage of Enhanced or Synthetic Vision Systems. Research discussed includes studies of field of view (FOV), representational differences of infrared (IR) imagery, head-up display (HUD) symbology, HUD advanced concept designs, sensor fusion, and sensor/database fusion and evaluation. Implications for the design and usage of Enhanced or Synthetic Vision Systems are discussed.
\end{abstract}

\section{INTRODUCTION}

Enhanced/Synthetic Vision is a term used to describe a group of advanced technology systems that will present or augment out-the-window information. Near-term designs (which we term Enhanced Vision Systems) propose presenting sensor imagery with superimposed flight symbology on a head-up display (HUD), and may include such enhancements as runway outlines and other display augmentations (e.g., obstacles, taxiways, flight corridors). Longer-term designs (which we term Synthetic Vision Systems) may include complete replacement of the out-the-window scene with a combination of electro-optical and/or sensor imagery and database information (as proposed in one version of the HighSpeed Civil Transport, HSCT). In these systems, the pilot would control the aircraft based on a representation of the world displayed in the cockpit, and may not see the actual outthe-window visual scene. Such systems present visual information that is needed but would not otherwise be visible (e.g., increased runway visibility in poor weather). It is likely, however, that some visual information will be lost, due to such limitations as resolution, field of view, or spectral sensitivities. Clearly, the most important and salient visual cues for pilotage must be maintained in the display. With Enhanced or Synthetic Vision Systems, the pilot no longer views the world directly; but views a representation through sensors and/or computerized databases. In these cases, it is important to determine the extent to which the Enhanced/Synthetic Vision System accurately transduces or represents the visual cues that impact flight control or taxi with such systems. If visual cues required for pilotage are not accurately or reliably represented to the pilot, system performance may suffer and safety could be compromised.

Some specific studies from current research in the Aerospace Human Factors Research Division at NASA Ames Research Center related to Enhanced/Synthetic Vision System design or usage are presented herein. NASA Ames Research Center and the other NASA centers have a long history of engineering and human factors research related to these topics. The discussion in this paper is limited to the work in the Aerospace Human Factors Research Division at NASA Ames Research Center. Research topics to be discussed include: Studies of head-up display (HUD) symbology and concepts, field of view (FOV), infrared (IR) imagery, and sensor fusion algorithms and evaluation.

\section{CONVENTIONAL HEAD-UP DISPLAYS (HUD)}

FLIGHT GUIDANCE INFORMATION -- Forty years ago, there were two separate sources of flight information: the panel instruments and the out-the-window scene. Gyroscopic instruments and precision ground-based radio navigation aids were developed to replace the out-thewindow information during Instrument Meteorological Conditions (IMC). One such radio aid for precision instrument approaches is the Instrument Landing System (ILS). The ILS consists of two radio beams that present lateral (localizer) and vertical (glideslope) course guidance information. The primary goal of a pilot flying an ILS approach is to keep the aircraft centered on this course until a predetermined altitude, or decision height (DH) is reached. At this point, the pilot must land the aircraft using visual cues (if they are available), or execute a missed approach. The ILS display that the pilot uses typically consists of two "crosshairs", which the pilot attempts to keep in a null, or centered, position. Deviation of the crosshairs from the center position indicate an angular offset of the aircraft from the desired course.

Pilots flying small aircraft find executing an ILS approach to be a demanding, but achievable task. In larger aircraft, which typically fly at faster approach speeds and are 


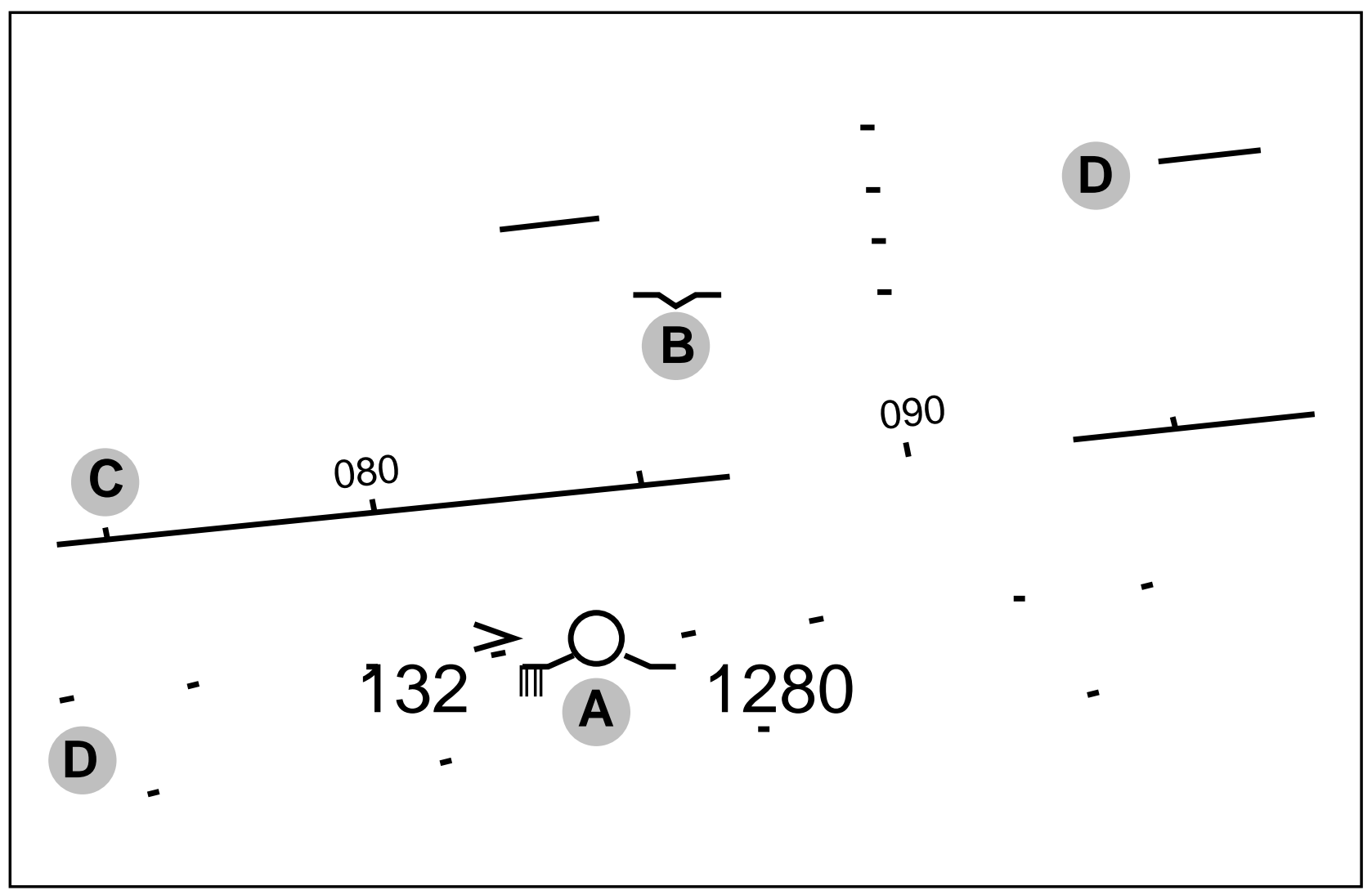

Figure 1. Schematic diagram of flightpath HUD (after Bray, 1980). Specific features include: A) flightpath (circle with "wings"), digital indications of airspeed (left) and altitude (right); B) aircraft attitude reference symbol; C) conformal horizon with heading markers; and, D) pitch attitude references (dashed lines). The flight condition portrayed is a slight right bank, with a heading of $087^{\circ}$, track angle of $084^{\circ}$. and reference heading of $090^{\circ}$. The pitch attitude is approximately $3^{\circ}$ above the horizon and the flightpath is $3^{\circ}$ below.

less maneuverable than small aircraft, the task of performing an ILS approach with only standard instrumentation and ILS deviations becomes extremely difficult. One system developed to aid the pilot in performing the task is the flight director. In a flight director, the ILS signals are processed by a computer, which calculates a trajectory which will allow the aircraft to intercept and track the ILS course while remaining within certain aircraft performance limits. The flight director then calculates the required roll and pitch for the aircraft to track the trajectory. These changing roll and pitch references are presented to the pilot on the Attitude Director Indicator (ADI), and the pilot flies this computed trajectory by nulling some type of error indication. Instead of directly "zeroing" errors in course, the pilot is assigned the task of zeroing pitch and roll errors, while another system assures that the course error is nulled. Although a well-designed flight director can make the ILS approach task much easier, it does not lend itself well to maintaining awareness of the current aircraft state, because it does not give a direct indication of course offset.

HUD SYMBOLOGY -- Today's technology has blurred the separation between panel instruments and out-thewindow scenes as sources of flight information. Superimposed flight symbology, whether on a HUD or a panel- or head-mounted display of sensor imagery now allows a level of integration that was not possible previously. The HUD naturally lends itself to the presentation of conformal information. Instead of using the panel-mounted ADI ball for attitude information, the HUD allows presentation of attitude information with an artificial horizon which is conformal with the outside scene, and with additional pitch and heading references. The HUD is also ideally suited for the presentation of flightpath information (the actual direction of flight, as opposed to orientation of the aircraft). The flightpath is also sometimes referred to as the velocity vector of the aircraft. Without a direct presentation of flightpath, the pilot is required to do visual scanning and mental transformations to determine the path of the aircraft. A display featuring flightpath information allows for a more natural, or intuitive method of control. Since the flightpath indicates directly where the aircraft is going, the pilot can assure landing on the runway by visually aligning the flightpath symbol with the end of the runway. Similarly, the pilot can track the ILS course by simply placing the flightpath symbol onto the course (if the course if displayed in a conformal manner), as opposed to following the trajectory commands of a flight director. In the flightpath display, the pilot can determine what trajectory to use to intercept the course. An early example of a HUD using these advanced features is described as follows (Lauber, Bray, Harrison, Hemingway \& Scott, 1982; Bray, 1980).

A joint program was undertaken in 1977 between NASA Ames Research Center and the Federal Aviation Administration (FAA) to evaluate the use of head-up displays 


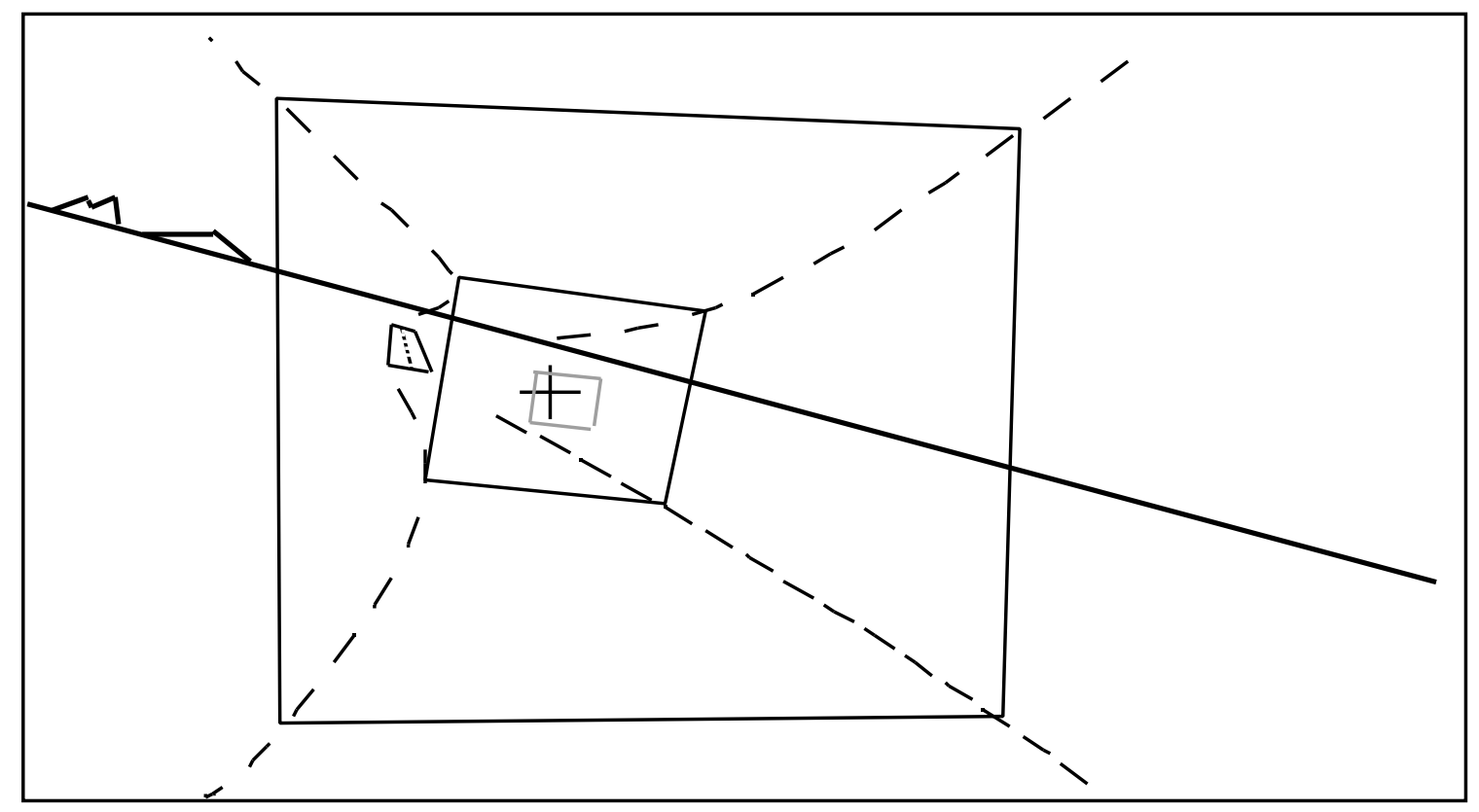

Figure 2. Schematic of a perspective pathway-in-the-sky display, showing curved approach into runway.

in commercial transport operations. NASA Ames had major responsibility for evaluation of human factors issues, and development and testing of candidate HUD formats. Two types of HUD formats were tested: a flight-director (FD) HUD, and a flightpath (FP) HUD. With only minor variations, the FD HUD presented the same information to the pilot as the head-down flight-director. The FP HUD was a conformal, earth-referenced display which portrayed the predicted flightpath of the aircraft, as well as navigation and aircraft state information (see Figure 1). In simulation studies (based upon Boeing 727 flight characteristics) conducted at Ames with line-captains from nine airlines, the FP HUD was rated superior to the head-down instruments for all cases. The FD HUD was generally not rated significantly different from the head-down condition.

This FP HUD display concept was later adopted and certified for use in the Boeing 727 aircraft by an avionics company. The new design incorporated a flight director element, while still featuring the flightpath symbology in the original display. Using this system, landings can be made in Category IIIa conditions (50 ft DH, $600 \mathrm{ft}$ RVR), with the pilot hand-flying the approach throughout the landing. Other conventional panel-mounted avionics systems (including conventional flight directors) require landings to be made by an autoland system (in which an automated system guides the aircraft, as opposed to the pilot) in Category IIIa conditions.

Although the utility of a HUD displaying flightpath information was clearly demonstrated, it should be noted that the flightpath display was based upon predicted, as opposed to instantaneous or current, information (Bray \& Scott, 1981). Because of the natural dynamic response of the B727 aircraft, the flightpath of the aircraft typically lags a pitch attitude change by 1.5 seconds at approach speeds. Precise control of flightpath is difficult because of the lag, since the flight controls the pilot is using directly affect pitch attitude, not flightpath. In the FP HUD design, the flightpath symbol term was compensated with a pitch attitude "lead" term. The change in pitch attitude was used to predict the flightpath change, and quicken the response of the flightpath symbol. McRuer and Krendal (1974) have shown that such display compensation is often necessary to achieve acceptable performance in human manual control tasks, depending upon the dynamic qualities of the system being controlled.

SUPERIMPOSED SYMBOLOGY FIXATION -Superimposed symbology, whether on a HUD or HMD, under certain conditions, has been demonstrated to lead to visual and attentional fixation. Under visual fixation, pilots are less likely to process other symbology information, and/or the world seen through the HUD or the imagery presented on the HUD (Fischer, Haines \& Price, 1980). Foyle, Sanford and McCann (1991) demonstrated that this fixation may be due to attentional issues rather than to visual factors (i.e., misaccommodation), as suggested by Iavecchia, Iavecchia and Roscoe (1988). Foyle et al also found that when augmented information is integrated into the visual scene, it does not suffer from the same attentional fixation problems that it does when presented through superimposed symbology. This suggests that using augmentations to the visual scene may mitigate the problem of visual/attentional fixation, as discussed below.

\section{SCENE AUGMENTATION}

One design challenge that faces the human factors and engineering communities is to design visual displays that preserve the most useful and unambiguous visual cues pilots naturally use. This can be accomplished through the development of designs that augment or enhance those visual cues. Through augmentation of the visual out-the-window scene under reduced-visibility conditions or through sensor use, the pilot can use these new, augmented cues in place of the missing or degraded cues available under better visual 


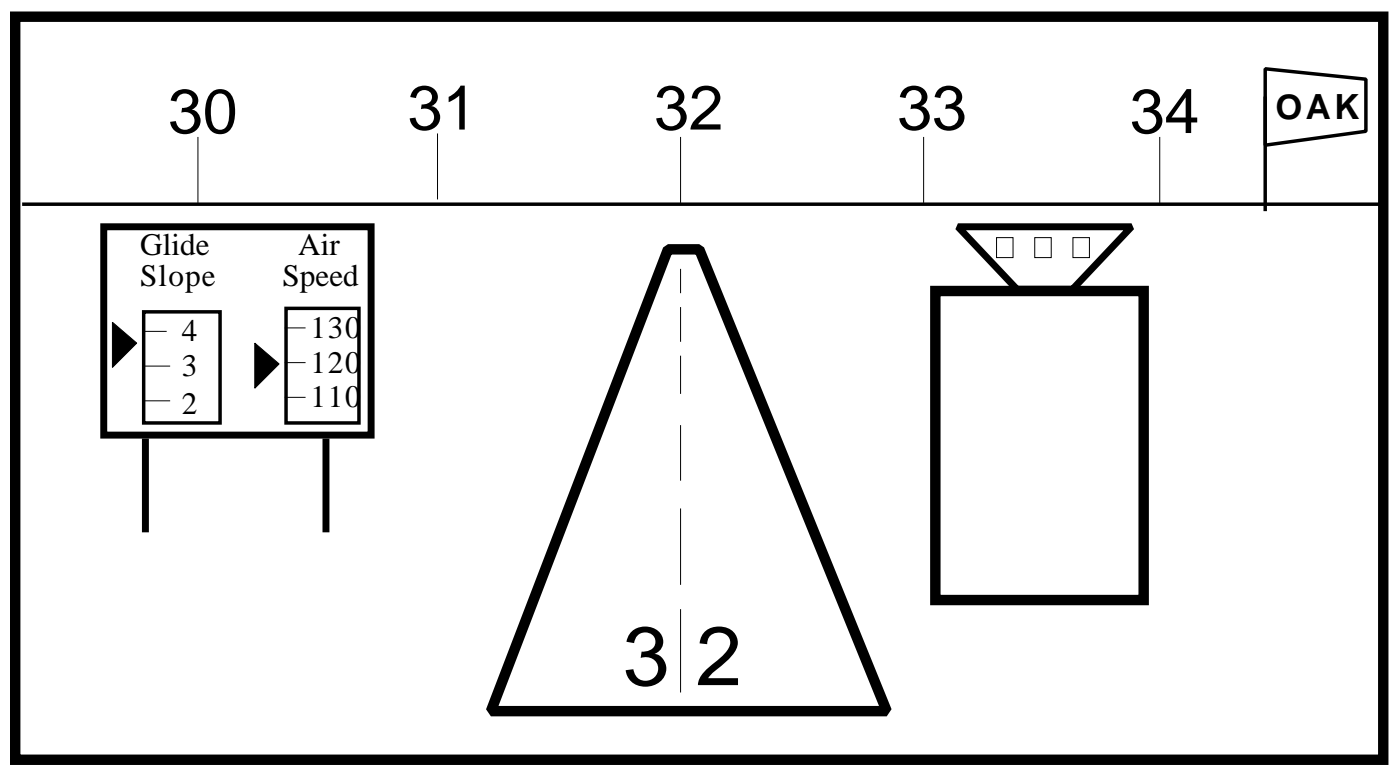

Figure 3. Schematic of a runway landing area demonstrating the "scene-linked HUD display" concept. The tower and runway represent real objects in the out-the-window scene. The compass rose attached to the horizon, distant airport (OAK) pennant and the billboard with instrument displays represent virtual, computer-generated images.

conditions. In the near-term, this augmentation may be done through HUD symbology, or, more practically and with more natural representations, in the long-term, in an Enhanced/Synthetic Vision System. Some examples of these augmentations range from the addition of a conformal horizon line, image processing to increase contrast (e.g., of runways), enhancing subthreshold information (e.g., distant runways, optical flow information), all the way to "making the invisible visible," such as showing graphically and spatially wind shear zones or taxiways and flight paths. Following are two candidate examples of scene augmentation for Enhanced/Synthetic Vision Systems.

PATHWAY-IN-THE-SKY DISPLAYS -- Grunwald and his colleagues (Grunwald, 1984; Dorighi, Ellis \& Grunwald, 1991) have designed a perspective display that shows the pilot the location of the aircraft relative to a planned or assigned flight path. This display shows current flight path status, but also displays predictive information such as future position and aircraft attitude, coupled with future path and bank angle. One candidate pathway-in-the-sky design is a wire-frame tunnel as shown in Figure 2. Coupled with highly accurate positioning systems such as Global Positioning System (GPS) or a precision-landing aid such as the Microwave Landing System (MLS) this pathway-in-the-sky display could be presented on existing HUDs to show assigned or predicted flight path information. Dorighi et al have argued that such displays are more natural and more efficient to use under good visibility conditions, and that under low-visibility conditions and high workload they may result in increased awareness of ground features and path position. They have also suggested it as a candidate display for the presentation of assigned curved approaches for these reasons.

SCENE-LINKED HUD DISPLAYS -- Advanced display media such as HUDs, in combination with highly accurate positioning systems allow for the possibility of placing information into the visual scene and stabilizing it with respect to the out-the-window scene. On the basis of the results of Foyle, Sanford and McCann (1991), this could allow for the viewing of the displayed information with reduced attentional problems mentioned above. That is, such a display may allow for the pilot to process visually both the displayed information and the out-the-window information without fixation or large attentional switching delays.

Figure 3 shows an example of such a scene-linked display. In the figure, the tower and runway represent actual items in the out-the-window image (either viewed through the HUD, or via sensor imagery on the HUD). The compass rose attached to the horizon line, and the pennant indicating a distant airport (OAK) at that location, represent virtual, computer-generated imagery that is drawn as if it were "attached" to the image or world. Likewise, the Glideslope/Air Speed instruments are displayed on a virtual, computer-generated billboard, visually projected as if placed to the side of the runway alongside a nominal aimpoint. HUD symbology is already in use that presents compass rose/horizon line information in this manner. Benefits, in addition to that of decreasing attentional problems, may occur from augmenting the visual scene in this manner. The addition of items of known size and consistent location allows the pilot to use the scene-linked display as a reference, using pictorial relationships. For example, the billboard could be constructed so as to appear to have a height equal to the decision height for landing. Adding this redundant pictorial and perspective cue could allow quicker processing and lower workload for altitude assessment. The visual flow field would be augmented as well by the scene-linked augmentations. For example, the virtual displays (e.g., billboard) would grow larger as one approached the runway, and any pitch or yaw of the aircraft would be perceived incidentally when viewing the display values. Research is underway to investigate the 
usefulness of the concept of scene-linked displays for augmenting the visual scene.

\section{SENSOR SYSTEM CHARACTERISTICS}

New sensor systems in the near future will undoubtedly have relatively narrow field of views (FOV), along with other limitations (possibly low spatial and temporal resolution, as well as other specific attributes). In addition, these sensor systems have an important (from a human factors view) inherent characteristic: These sensors all generate visible images created from a representation of the world in a spectral band, with which the pilot has no experience. Following are two studies related to these sensor system characteristics.

FIELD OF VIEW -- Brickner and Foyle (1990) conducted a simulation of a helicopter slalom course flight task with a forward-looking imaging sensor. Three FOV values were tested: 25,40 and $55 \mathrm{deg}$. Slalom course performance was measured by tallying number of slalom course pylon hits and averaging altitude and course deviations. Not surprisingly, the data indicated that flight control was best (and approximately equal) in the 40 and $55 \mathrm{deg}$ FOV conditions, with an increased number of pylon hits occurred in the 25 deg FOV condition. An unexpected result was obtained when the flight paths were analyzed: The turns around the pylons were closest to the pylons in the $25 \mathrm{deg}$ FOV condition, and largest for $55 \mathrm{deg}$ FOV. This was explained by suggesting that the subject pilots adopted a flight control strategy that was greatly influenced by the FOV: Subject pilots may have attempted to maintain the image of the pylons at the edge of the visible display, simultaneously maximizing aircraft distance while maintaining sight of the pylons. For Enhanced/Synthetic Vision Systems, especially if used for taxi in fog, these data may indicate a tendency to adjust aircraft ground track to keep obstructions visible on the display. In general, these data indicate that there may be very subtle, surprising effects of FOV on control. For Synthetic Vision Systems, one design that could overcome this problem is to inset, using sensor fusion techniques, a narrow FOV sensor into a graphical database image, effectively increasing the system FOV.

INFRARED (IR) IMAGERY -- Infrared imagery transduces thermal energy into a visible image on a display. These sensors have been used by the military and others for night flight, since they are do not require any visible light to produce imagery. There are numerous differences between the images presented by these systems and the normal visual scene viewed during normal daytime flight, each having a consequence on flight performance. These include displaydevice and sensor-related factors (e.g., monocular imagery, FOV restrictions, spatial and temporal resolution), and the inherent perceptual difficulties associated with flight based on imagery generated from thermal differences rather than from reflected visible light.

Foyle, Brickner, Sanford and Staveland (1990) conducted a study in which subjects identified terrain-type targets (e.g., trees, canyons) and non-terrain type targets (e.g., roads, vehicles) with color television (TV) and IR imagery. As can be seen in Figure 4, the recognition of objects in general is greatly affected by the type of imagery viewed.
Non-terrain objects were recognized faster with IR imagery than when viewed with television. The terrain targets, however, were recognized faster with television imagery. An analysis of the display parameters (i.e., contrast, luminance) associated with the targets indicated that for the non-terrain objects, the difference in recognition time varied as a function of the display parameters. That is, for the non-terrain objects, higher contrast and luminance levels were associated with faster recognition time. Presumably the unnaturalness of the gray-shade mapping found in IR imagery was mitigated by the increased visibility due to contrast and luminance. For the terrain targets, recognition time was not related to the display parameters, and it was hypothesized that recognition performance of terrain targets was more influenced by cognitive factors. That is, under IR imagery, the terrain targets did not appear as expected. Additionally, in a separate study, the specific gray-shade mapping for IR non-terrain targets has also been found to influence recognition. Brickner and Zvuloni (1991), found that IR polarity, the mapping of black to hot or white to hot, strongly affected the ability to recognize non-terrain objects. Thus, for non-terrain targets, it appears that IR imagery improves recognition performance over direct vision or TV (due to display parameters), but that the gray-shade mapping can also affect recognition.

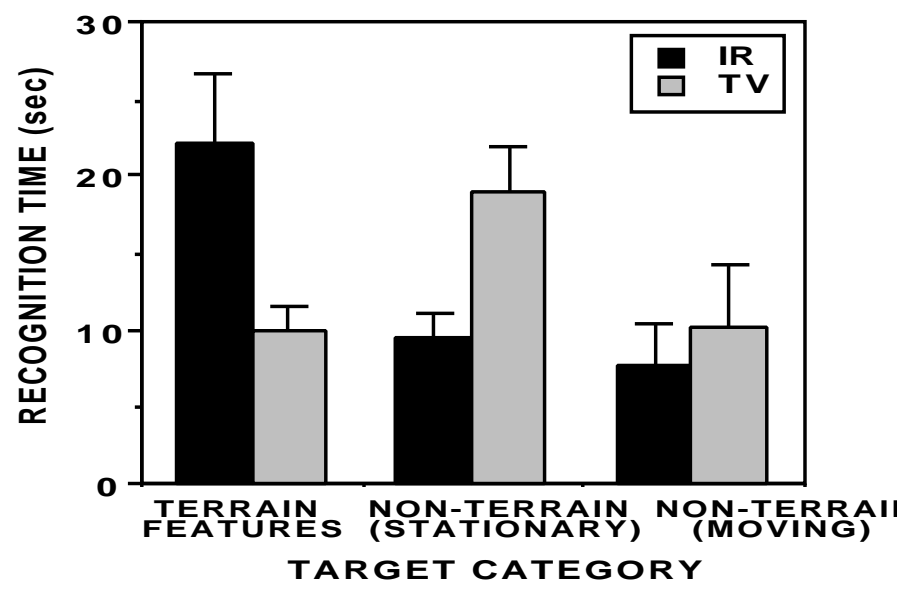

Figure 4. Mean recognition time for terrain and nonterrain objects viewed with television (TV) or infrared (IR) imagery.

This inherent difference between the appearance of IR imagery and direct vision or TV may have impact on the use of Enhanced/Synthetic Vision Systems. Runways and taxiways may have a quite different appearance under IR imagery (and presumably other non-visible wavelength passive imaging systems, such as passive millimeter wave, PMMW). The visual cues in runways and taxiways normally present with direct vision may either be augmented or degraded depending on environmental conditions and material makeup. For example, an IR sensor may or may not image the 


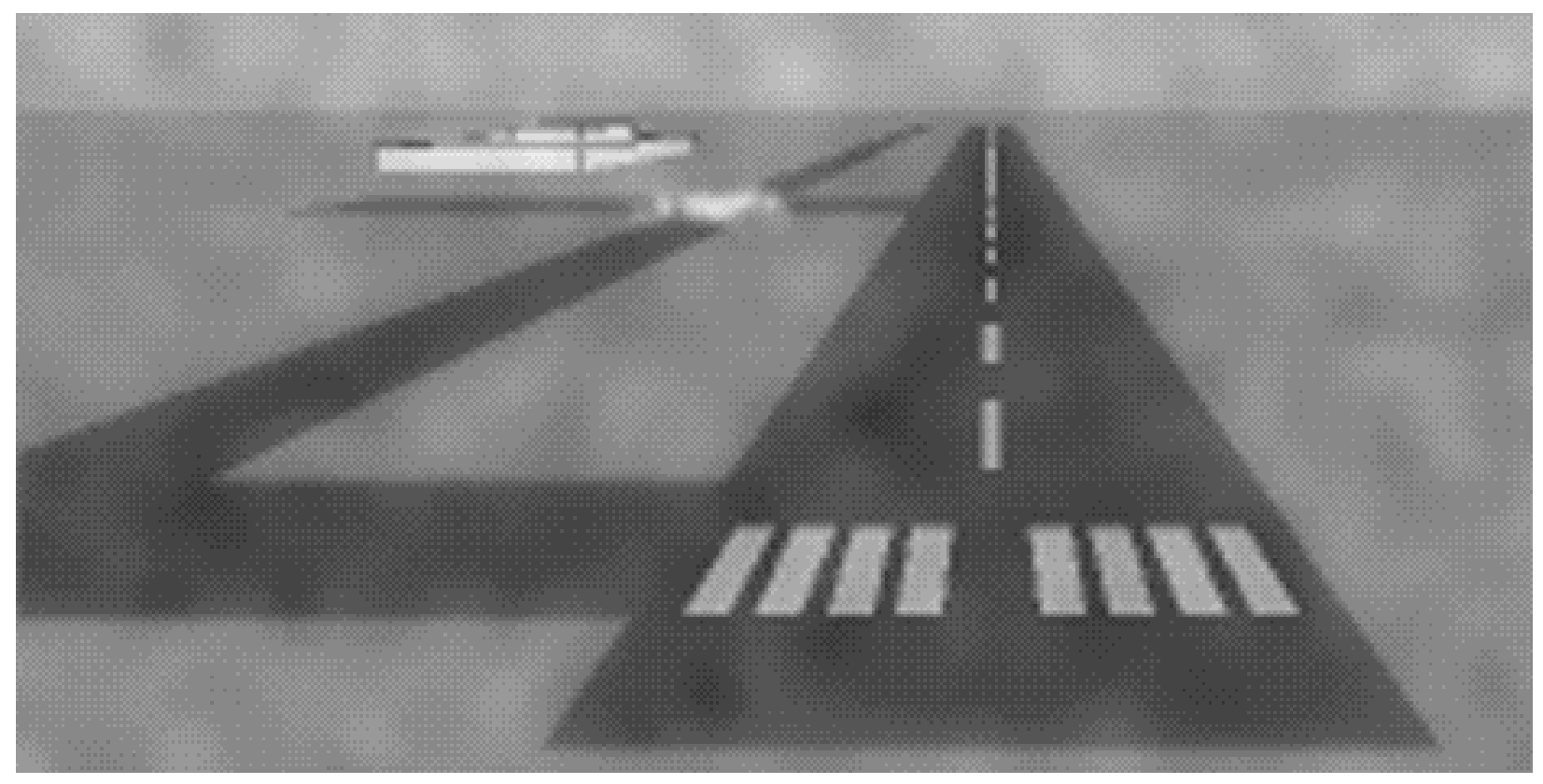

Figure 5. Simulated sensor fusion image resulting from fusion of graphical database image and PMMW sensor.

runway paint markings, dependent upon the thermal history, and the emissive and reflective properties. Objects or features that have low contrast in the visible spectrum may have high contrast in the thermal image, such that "artifactual" characteristics may be accentuated, such as expansion joints and filled cracks in the runway. The impact that these inherent differences in the visual representation have on pilot performance is not known and clearly needs to be assessed.

\section{SENSOR FUSION}

The goal of sensor fusion algorithms is to combine different sources of information into a single display, both to reduce the number of displays required and to reduce the workload of the pilot attempting to integrate rapidly the information from the different sources. Here we briefly describe an approach to sensor fusion described in more detail elsewhere (Pavel, Larimer \& Ahumada, 1991; 1992). Although the approach is generalizable to other sensors and other tasks, this discussion will focus on the task of deciding whether to land or execute a missed-approach in low visibility based on the information from an active or passive millimeter wave sensor and from position information that allows an image of the scene to be rendered from a graphical data base of the terminal environment. The combining rule will be based on relatively simple properties of the images. This approach does not perform scene analysis or try to use the sensor information to infer the state of the world, as other, more sophisticated methods do (Hager, 1990; Clark \& Yuille, 1990). Our goal is to produce a fusion algorithm that can be implemented using known methods and available hardware (van der Wal, 1991).

This effort focuses on two major problems of radar images, spatial resolution limitations caused by the limited antenna size and noise limitations caused by signal power limitations in conjunction with intrinsic sensor noise. The major limitation of the position-based (data-based rendered) image is that it does not contain time-varying obstacles, such as other aircraft or ground vehicles that may be on or headed onto the runway. Two major problems are not yet addressed: image contrast direction incompatibilities and image alignment problems. In the near-term, presumably, it will be easier to render the database as a long-wavelength image than it will be to transform a millimeter wave (MMW) or IR image into a visual image of the same scene despite the attendant problems of interpretation (e.g., Foyle, Brickner, Staveland \& Sanford, 1990). The image registration problem has been shown to be simplified by the multiresolution image analysis used in the fusion (Burt \& Adelson, 1983). The approach to solving the problems is based on two principles, multiresolution analysis and noise analysis.

MULTIRESOLUTION IMAGE ANALYSIS -Noting the success of Toet and his colleagues (Toet, van Ruyven, \& Valeton, 1989; Toet, 1989; 1990; 1992) in fusing visual and IR imagery, we have taken the multiresolution image analysis approach pioneered by Burt and Adelson (Burt \& Adelson, 1985; Burt, 1984; 1992). In this approach the image is first decomposed into bandpassed component images in a manner analogous to the generation of separate bandpassed audio signals by a stereo system graphic equalizer. The fusion algorithm combines two corresponding bandpass component images, one from each source, and then these fused bandpass component images are combined. There are two major benefits from this approach. One benefit is that the high-spatial resolution image component of the position-based image is not subjected to the fusion algorithm at all. Only the low-resolution part of the position-based image, which is represented by the same small number of image values as the low-spatial resolution radar image, participates in the combination. This results in large computational savings. This ability of the multiresolution approach to match images of different resolutions also allows it to work easily with 
variable resolution sensor data. A second advantage is that combination of images in the different spatial frequency bands results in little cross-masking between the different bands. Information in one band can be seen relatively transparently through the other bands. This may explain in part the success of the fusion of visual and IR imagery despite the lack of any concern for contrast polarity.

NOISE ANALYSIS -- Much of the total contrast energy of low-signal, high-noise image is just noise. Pavel, Larimer, and Ahumada (1992) have proposed maintaining a running estimate of the noise level at each sensor region and resolution, so that only the statistically significant components of the noisy image are selected for output. If the signal energy of a region of a bandpass image is in the noise level, the corresponding component of the position-based image is selected. Since the radar image has no signal energy at highspatial frequencies, these bands are determined only by the position-based image.

SENSOR FUSION IMAGE -- Figure 5 shows the result of fusing a simulated PMMW image with the corresponding graphical database image. In this example, the PMMW sensor has imaged the scene with an aircraft taxiing towards the runway during approach. The position-based image has the same scene information without the obstacle. In the fused image, the intruding aircraft is essentially as visible as it was in the original sensor image, although it is lacking the detail of objects that are also in the database. Hopefully, the pilot seeing such a blob approaching the runway would decide to go around.

EVALUATION -- Although this sensor simulation ignores the difficulties the viewer may have because of the long-wavelength radiation issues and registration problems, simulation of the noise and resolution effects on a visual image allows bounds to be placed on allowable values for those parameters. More realistic simulation would only degrade performance. These images demonstrate that modest workstations can support the calculation of images that could be used for psychophysical evaluation of possible sensor fusion schemes. Computational evaluation of the detectability of obstacles in such scenes would allow the system designers to search a much larger set of possible systems than it would be practical to evaluate psychophysically. Because they must separate components of the scene according to how they mask each other, models of human visual target detection often contain a stage of bandpass filtering similar to those of the fusion method (Wilson \& Bergen, 1979; Carlson \& Cohen, 1980; Watson, 1983). Although the state of the art in such modeling is not such that accurate $a$ priori predictions of such masking are possible, if such models were calibrated on psychophysical measurements of appropriate target detection performance, they would allow accurate interpolation and reasonable extrapolation of the psychophysics.

Based on existing detection-based information integration models in the literature, Foyle (1992) developed an evaluation framework to assess an operator's ability to use multisensor, or sensor fusion, displays. The proposed framework for evaluating the operator's ability to use such systems is a normative approach: The operator's performance with the sensor fusion display can be compared to the models' predictions based on the operator's performance when viewing the original sensor displays prior to fusion. This allows for the determination as to when a sensor fusion system leads to: 1) poorer performance than one of the original sensor displays (clearly an undesirable system in which the fused sensor system causes some distortion or interference); 2) better performance than with either single sensor system alone, but at a sub-optimal (compared to the model predictions) level; 3) optimal performance (compared to model predictions); or, 4) super-optimal performance, which may occur if the operator were able to use some highly diagnostic "emergent features" in the sensor fusion display, which were unavailable in the original sensor displays. The approach is a "detectionoriented" approach in which predicted detection performance with the sensor fusion display is compared to actual detection performance. Detection performance is a relevant task measure for certain aspects of landing with Enhanced or Synthetic Vision Systems. The visibility of runway incursions or obstacles, clearly can be characterized by detection performance. Likewise, in order to proceed with a landing, at decision height, the FAA requires the detection of any of ten separate runway features (e.g., runway lights, threshold markings, etc., see FAA FAR Part 91.175-c). This evaluation framework can be used to determine if detection performance with the sensor fusion system is improved, and can determine the optimality of that improvement.

\section{SUMMARY}

This paper has reviewed some of the research related to Enhanced or Synthetic Vision Systems on-going in the Aerospace Human Factors Research Division at NASA Ames Research Center. One theme threaded through most of this work involves the enhancement through display augmentation of the visual out-the-window information. Out-the-window viewing in low-visibility weather or viewing sensor imagery with limited display characteristics may result in the degradation of the visual cues usually available. To counteract such degradation, advanced displays in which the out-the-window scene is enhanced or augmented are proposed. Such enhancements may add the necessary visual cues back to the scene, which were removed or made unreliable by the degraded operating conditions.

\section{ACKNOWLEDGMENTS}

We appreciate the assistance of M. Pavel and N. Dorighi. Supported by NASA RTOPS 505-64-36 and 505-64-53.

\section{REFERENCES}

Bray, R.S. (1980). A head-up display format for application to transport aircraft approach and landing, NASA TM 81199. Moffett Field, CA: NASA.

Bray, R.S. and Scott, B.S. (1981). A head-up display for low visibility approach and landing. Proceedings of the AIAA 19th Aerospace Sciences Meeting.

Brickner, M.S. and Foyle, D.C. (1990). Field-of-view effects on a simulated flight task with head-down and head-up 
sensor imagery displays. Proceedings of the Human Factors Society 34th Annual Meeting, 1567-1571.

Brickner, M.S. and Zvuloni, A. (1991). The effects of polarity on object recognition in thermal images. Unpublished manuscript. PAMAM Human Factors Ltd., Tel Aviv, Israel.

Burt, P. J. (1984). The pyramid as a structure for efficient computation. In A. Rosenfeld (Ed.), Multiresolution image processing and analysis. New York: SpringerVerlag.

Burt, P. J. (1992). A gradient pyramid basis for patternselective image fusion. SID International Symposium Digest of Technical Papers, 23, 467-470.

Burt, P.J. and Adelson, E.H. (1983). A multiresolution spline with application to image mosaics. ACM Transactions on Graphics, 2, 217-236.

Burt, P.J. and Adelson, E.H. (1985). Merging images through pattern decomposition. SPIE Proceedings 575, 173-181.

Carlson, C.R. and Cohen, R.W. (1980). A simple psychophysical model for predicting the visibility of displayed information. SID International Symposium Digest of Technical Papers, 11, 229-246.

Clark, J.J. and Yuille, A.L. (1990). Data fusion for sensor information processing systems. Boston: Kluwer.

Dorighi, N.S., Ellis, S.R. and Grunwald, A.J. (1991). Evaluation of perspective displays on pilot situational awareness in low visibility curved approaches. AIAA Computing in Aerospace 8: A Collection of Technical Papers, CP9110-1, 153-157.

Fischer, E., Haines, R.F., and Price, T.A. (1980). Cognitive issues in head-up displays. NASA TP 1711. Moffett Field, CA: NASA.

Foyle, D.C. (1992). Proposed evaluation framework for assessing operator performance with multisensor displays. Proceedings of the SPIE/IS\&T Symposium on Electronic Imaging Science and Technology, Conference 1666: Human Vision, Visual Processing, and Digital Display III.

Foyle, D.C., Brickner, M.S., Staveland, L.E. and Sanford, B.D. (1990). Human object recognition as a function of display parameters using television and infrared imagery. SID International Symposium Digest of Technical Papers, 21, 269-272.

Foyle, D.C., Sanford, B.D. and McCann, R.S. (1991). Attentional issues in superimposed flight symbology. In R.S. Jensen (Ed.), Proceedings of the Sixth International Symposium on Aviation Psychology, 577-582.
Grunwald, A.J. (1984). Tunnel display for four-dimensional fixed-wing approaches. Journal of Guidance, Control and Dynamics, 7, 369-377.

Hager, G.D. (1990). Task-directed sensor fusion and planning. Boston: Kluwer.

Iavecchia, J.H., Iavecchia, H.P., and Roscoe, S.N. Eye accommodation to head-up virtual images. Human Factors, 30, 689-702.

Lauber, J.K., Bray, R.S., Harrison, R.L., Hemingway, J.C. and Scott, B.C. (1982). An operational evaluation of head-up displays for civil transport operations. NASA TP 1815. Moffett Field, CA: NASA.

McRuer, D.T. and Krendal, E.S. (1974). Mathematical models of human pilot behavior. AGARDograph No. 188.

Pavel, M., Larimer, J., and Ahumada, A. (1991). Sensor fusion for synthetic vision. AIAA Computing in Aerospace 8: A Collection of Technical Papers, CP91101, 164-173.

Pavel, M., Larimer, J., and Ahumada, A. (1992). Sensor fusion for synthetic vision. SID International Symposium Digest of Technical Papers, 23, 475-478.

Toet, A. (1989). Image fusion by a ratio of low-pass pyramid. Pattern Recognition Letters, 9, 245-253.

Toet, A. (1990). Hierarchical image fusion. Machine Vision and Applications, 3, 1-11.

Toet, A. (1992). Multi-scale image fusion. SID International Symposium Digest of Technical Papers, 23, 471-474.

Toet, A., van Ruyven, L.J. and Valeton, J.M. (1989). Merging thermal and visual images by a contrast pyramid. Optical Engineering, 28, 789-792.

van der Wal, G. (1991). The Sarnoff pyramid chip. Proceedings CAMP-91.

Watson, A.B. (1983). Detection and recognition of simple spatial forms. In O. J. Braddick, A. C. Sleigh, (Eds.), Physical and biological processing of images. Berlin: Springer-Verlag.

Wilson, H.R. and Bergen, J.R. (1979). A four mechanism model for threshold spatial vision. Vision Research, 19, 19-32. 